\section{Epidemiologia da Leishmaniose \\ Tegumentar e descrição das populações de flebotomíneos no município de Acrelândia, Acre, Brasil}

\author{
Epidemiology of Cutaneous \\ Leishmaniasis and description of \\ phlebotomine sandfly populations \\ in the city of Acrelandia, Acre, Brazil
}

\section{Mônica da Silva-Nunes ${ }^{1}$ \\ Carlos Eugênio Cavasini² \\ Natal Santos da Silva ${ }^{1}$ \\ Eunice Aparecida Bianchi Galati ${ }^{3}$}

'Departamento de Parasitologia, Instituto de Ciências Biomédicas, Universidade de São Paulo

²Departamento de Doenças Dermatológicas, Infecciosas e Parasitárias, Faculdade de Medicina de São José do Rio Preto

${ }^{3}$ Departamento de Epidemiologia, Faculdade de Saúde Pública, Universidade de São Paulo

Fonte de financiamento: Fundação de Amparo à Pesquisa do Estado de São Paulo (Proc 02/04896-4).

Correspondência: Mônica da Silva-Nunes. Departamento de Parasitologia, Instituto de Ciências Biomédicas, Universidade de São Paulo, Av. Prof Lineu Prestes, 1374 - Cidade Universitária, CEP 05508-900 São Paulo, SP. E-mail: msnunes1@yahoo.com.br

\section{Resumo}

São apresentadas neste artigo a distribuição da leishmaniose tegumentar (LT) e descrição das populações de flebotomíneos em Acrelândia, Acre. Os dados epidemiológicos foram obtidos a partir de fichas de notificação de casos ocorridos entre 2001 e 2004, e os dados entomológicos são provenientes de capturas com armadilhas luminosas efetuadas entre 2004 e 2005 na zona rural de Acrelândia. Ocorreram 82 novos casos de LT, com idade entre 2 e 69 anos, sendo $75,6 \%$ em homens e $83,9 \%$ na zona rural. Predominou a LT com lesões únicas (78\%). A microscopia direta da lesão, intradermorreação de Montenegro e biópsia apresentaram positividade de $100 \%$, $98 \%$ e $79,5 \%$, respectivamente. A resposta ao tratamento farmacológico foi bem sucedida em $71,6 \%$ dos casos; a falência terapêutica foi maior em pacientes com diagnóstico exclusivamente clínico $(41,2 \%)$ e nos que receberam dose diária inadequada de antimonial pentavalente $(64,3 \%)$. Foram coletados 40 espécimes de flebotomíneos em propriedades rurais com casos de LT (3 gêneros, 14 espécies), sendo 3 espécies conhecidas como vetoras ou possíveis vetoras de Leishmania: Nyssomyia antunesi predominou no peridomicílio $(59,1 \%)$ e em margens de matas; Nyssomyia whitmani foi freqüente no peridomicílio (15\%) e a única espécie encontrada no intradomicílio, e Trichophoromyia ubiquitalis foi capturada no peridomicílio. O uso de dados epidemiológicos existentes no serviço de saúde de Acrelândia, embora com várias limitações, permitiu avaliar a eficácia do diagnóstico e o tratamento empregados no município, enquanto os dados entomológicos coletados podem orientar estudos mais amplos visando identificar os vetores e espécies circulantes na região.

Palavras-chave: Leishmaniose tegumentar. Flebotomíneos. Epidemiologia 


\section{Abstract}

This paper describes the distribution of cutaneous leishmaniasis (CL) in the city of Acrelandia, state of Acre, and its phlebotomine sandfly population. Epidemiological data were obtained from case reporting forms in 2001-2004, and entomological data resulted from catches performed between 2004 and 2005 using light traps. A total of 82 new cases of CL, aged between 2 and 62 years, $75.6 \%$ males, and $83.9 \%$ in the rural area, were identified. The prevailing clinical form was cutaneous $(92.7 \%)$ with a single lesion (78\%). Direct microscopic exams of lesions, Montenegro skin reaction tests, and biopsies resulted in 100\%, $98 \%$ and $79.5 \%$ positivity rates, respectively. The therapeutic response was successful in $81.7 \%$ of subjects; treatment refractoriness was higher among CL patients with clinical diagnosis only (41.2\%) and among those who received inadequate daily doses of antimonial drug (64.3\%). Forty specimens of phlebotomine sandfly were collected around houses with CL cases (3 genera, 14 species); three of those species are known as vectors or possible vectors of Leishmania: Nyssomyia antunesi prevailed in the peri-domiciliary environment (59.1\%) and forest borders; Nyssomyia whitmani was frequent in peridomiciliary areas (15\%) and was the only female captured in the intra-domiciliary environment; and Trichophoromyia ubiquitalis was found in the peri-domiciliary environment. The information derived from pre-existing epidemiological data, despite limitations, provided an evaluation of diagnosis and treatment efficacy, while the entomological data can be used as a guideline to develop a larger study aimed to identify both vectors and circulating Leishmania species.

Keywords: Cutaneous leishmaniasis. Phlebotomine Sandfly population. Epidemio$\log y$

\section{Introdução}

A ocorrência da leishmaniose tegumentar americana (LT) tem aumentado no Brasil nas últimas décadas, com 610.256 casos autóctones ${ }^{1}$ registrados entre 1980 e 2005, dos quais a região Norte contribuiu com $37 \%$. No entanto, há poucos estudos realizados na região amazônica, o mais importante foco de transmissão do país, com um coeficiente de detecção de 99,8/100.000 habitantes ${ }^{1}$. A expansão da LT na região amazônica parece estar relacionada a uma mudança de comportamento epidemiológico da doença, que antes limitada às zonas florestais, passou a ocorrer em zonas rurais desmatadas e peri-urbanas, facilitada pelo surgimento de áreas de garimpos, expansão de fronteiras agrícolas e formação de novos núcleos urbanos em ambientes favoráveis à transmissão da doença. Portanto, há a necessidade real de se caracterizar melhor os padrões epidemiológicos da LT na região amazônica, bem como seus possíveis vetores. Embora estudos prospectivos de campo sejam a melhor forma de avaliar as características epidemiológicas, $\mathrm{o}$ alto custo desse tipo de estudo e o tempo necessário para a coleta de dados acabam limitando a sua aplicabilidade. Por isso, a utilização de dados epidemiológicos provenientes dos próprios serviços de saúde, apesar de certas limitações, pode gerar informações importantes para a melhor compreensão da doença.

O Estado do Acre registrou crescimento importante do número de casos nos últimos anos, sendo que a prevalência de LT aumentou de 9,3 casos / 10.000 habitantes ${ }^{2}$ para 18,6 casos/10.000 habitantes entre 1992 e 2002 (Gerência de Vigilância Ambiental do Acre, dados não publicados). A maior parte desses casos está concentrada na microrregião de Rio Branco $^{2}$, da qual faz parte o município de Acrelândia. Há poucos dados disponíveis sobre potenciais vetores de LT no Acre e na região amazônica como um todo ${ }^{3,4}$. A presença de N. whitmani sensu lato já foi descrita na Amazônia ${ }^{5}$, sendo comprovadamente transmissora 
de L. (V.) shawi no Pará6,7; entretanto, seu papel na transmissão de Le. braziliensis nessa parte do país ainda é discutido ${ }^{8}$, uma vez que parece ser pouco antropofílica na Amazônia ${ }^{9}$. Outra espécie antropofílica associada à transmissão da LT e encontrada no Amazonas e no Acre é a N. umbratillis (Ward \& Fraiha, 1977) ${ }^{5,10-12}$. Esses dados, no entanto, são generalizados para toda Amazônia e não há estudos recentes particularizando a fauna de flebotomíneos no Acre, em áreas de transmissão de LT.

Os objetivos deste estudo são descrever as características epidemiológicas da leishmaniose tegumentar notificada em Acrelândia, Acre, no período de janeiro de 2001 a dezembro de 2004, e realizar um levantamento preliminar da fauna flebotomínica na zona rural do município, para identificar possíveis vetores da doença na região.

\section{Metodologia}

\section{Área e população de estudo}

Acrelândia tem população de 8.697 habitantes ${ }^{13} \mathrm{e}$ área de $1.607,5 \mathrm{~km}^{2}$, sendo que cerca de metade da população reside na zona rural. Situa-se a $112 \mathrm{~km}$ a leste de Rio Branco (capital do Estado do Acre), fazendo fronteira com os municípios acreanos de Senador Guiomard e Plácido de Castro, os Estados do Amazonas e Rondônia e a Bolívia (Figura 1). O solo predominante é o argissolo amarelo, e o clima é equatorial, quente e úmido. Apresenta uma estação chuvosa entre novembro e abril, e uma estação seca entre maio e outubro. A temperatura média anual é de $24,5^{\circ} \mathrm{C}$, variando entre $20^{\circ} \mathrm{C}$ e $32^{\circ} \mathrm{C}$; a umidade relativa do ar é de $80-90 \%$ durante o ano todo. Os índices pluviométricos anuais estão entre $1.600 \mathrm{~mm}$ e $2.750 \mathrm{~mm}$; entretanto, a contínua devastação da paisagem nativa tem alterado o regime das chuvas nos últimos anos. A vegetação nativa da região é a floresta ombrófila densa das terras baixas que, em virtude dos assentamentos dirigidos, áreas de invasões e exploração de madeira de lei, têm sofrido profundas mo- dificações ${ }^{14}$. O índice de desenvolvimento humano (IDH) estimado em 2000 para a população geral do município é de $0,680^{15}$, com uma taxa de mortalidade infantil de 24,69 por 1.000 nascidos vivos (Datasus, $2005)^{13}$. A taxa de analfabetismo é de $26,7 \%$, chegando a $31,9 \%$ na zona rural ${ }^{15}$, onde vivem $57 \%$ dos habitantes do município. O município de Acrelândia alberga vários projetos de assentamentos rurais dirigidos pelo Instituto Nacional de Colonização e Reforma Agrária (INCRA), sendo o maior deles o Projeto de Assentamento Dirigido (PAD) Pedro Peixoto (Figura 1), instituído em 1977.

O diagnóstico de leishmaniose é realizado na Unidade Básica de Saúde central (UBSc), localizada na zona urbana do município. Os casos confirmados laboratorialmente e/ou epidemiologicamente são encaminhados para tratamento e investigação complementar pela equipe do Serviço deVigilância Epidemiológica de Acrelândia (SVEA). Além disso, a Secretaria Estadual de Saúde do Acre (SESSACRE) mantém equipes volantes de vigilância ambiental na zona rural, que encaminham casos suspeitos para a UBSc.

\section{Coleta de variáveis epidemiológicas}

As informações dos casos de LT em indivíduos domiciliados no município de Acrelândia e notificados entre janeiro de 2001 e dezembro de 2004 foram obtidas no SVEA, em consulta às fichas de notificação de casos de leishmaniose distribuídas pelo Ministério da Saúde (MS) e utilizadas na unidade notificadora. Foram analisadas as seguintes variáveis: sexo, idade, profissão, local de moradia (rural ou urbano), tipo de lesão (cutânea, mucosa, cutâneo-mucosa e cutânea difusa), métodos diagnósticos (exame microscópico do raspado da lesão, reação intradérmica de Montenegro, biópsia ou exame clínico apenas), forma clínica (cutânea ou mucosa), tipo de entrada (caso novo ou recidiva), tratamento utilizado (tipo de fármaco e dose diária prescrita), adequação da dose diária de antimonial pentavalente prescrita 


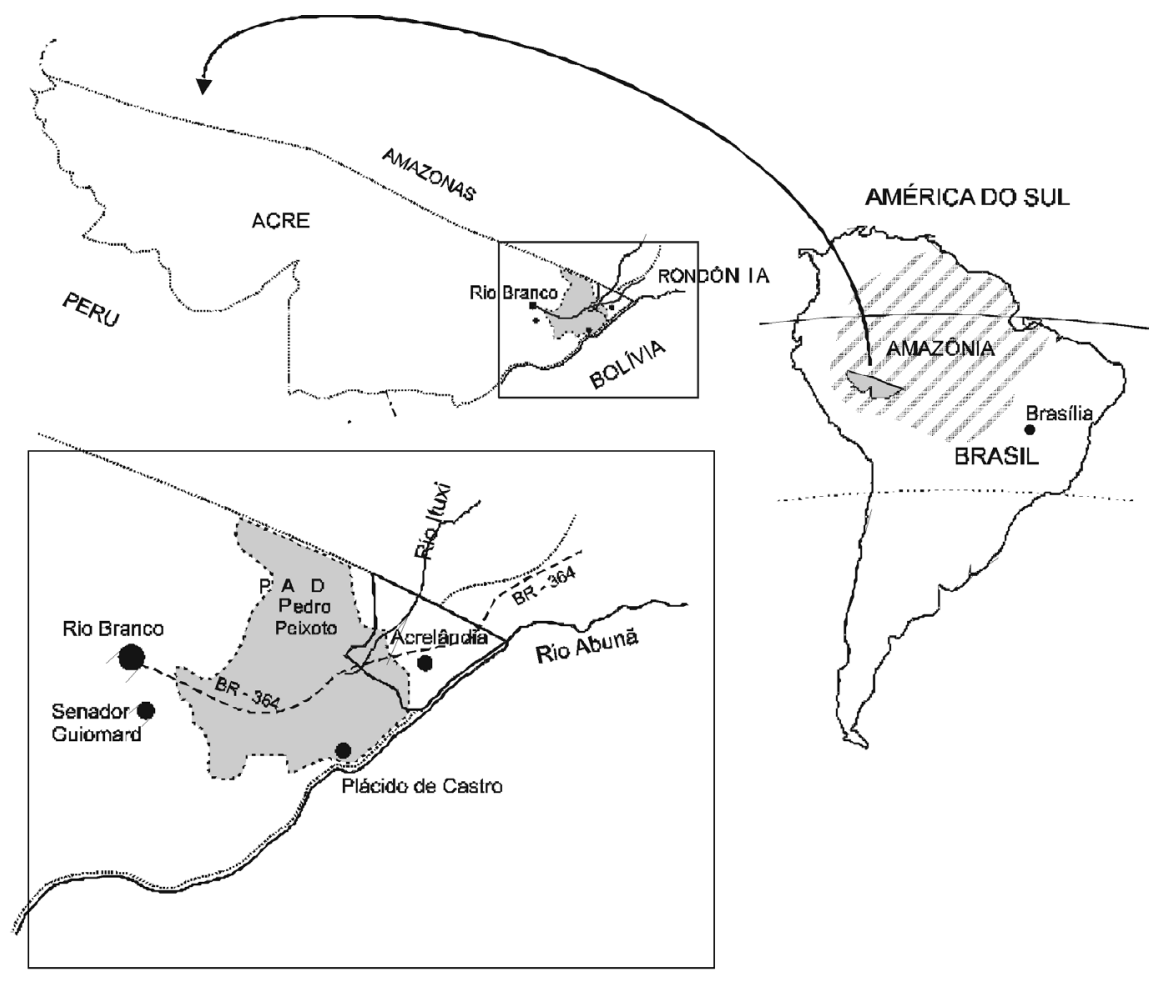

Figura 1 - Mapa da América do Sul mostrando a região Amazônica, o Estado do Acre, a localização do município de Acrelândia e do Projeto de Assentamento Dirigido Pedro Peixoto (PAD Pedro Peixoto). O ramal do Granada, local onde foram realizadas as capturas de flebotomíneos, localiza-se dentro do PAD Pedro Peixoto.

Figure 1 - Map of South America showing the Amazon, state of Acre, city of Acrelandia and PAD Pedro Peixoto. Ramal do Granada, where phlebotomines were captured, is located inside PAD Pedro Peixoto.

segundo as recomendações do Ministério da Saúde (adequada ou inadequada; doses recomendadas de 10 a $20 \mathrm{mg} / \mathrm{kg} /$ dia para formas cutâneas; $20 \mathrm{mg} / \mathrm{kg} /$ dia para formas cutâneas difusas e qualquer forma mucosa) ${ }^{16}$ e resposta à terapia farmacológica (sucesso se houve remissão completa das lesões em até três meses do término do tratamento, $\mathrm{e}$ falência se não houve remissão das lesões). As definições utilizadas são aquelas constantes da ficha de notificação do Sistema de Informação de Agravos de Notificação entre 2001 e 2004, publicadas no Manual de Vigilância da Leishmaniose Tegumentar Americana ${ }^{16} \mathrm{e}$ em Gontijo \& Carvalho (2003) ${ }^{17}$.

\section{Pesquisa de Flebotomíneos}

A área de coleta de flebotomíneos compreende as localidades rurais conhecidas como Linha 14 Gleba Q e Reserva da Linha 14 , onde se concentra a maioria dos casos recentes de LT do município. Essas duas localidades possuem, em conjunto, população de 466 indivíduos distribuída em 113 domicílios (dados de 2004) ${ }^{18}$, o que representa um adensamento populacional relativamente grande em área agrícola recente com grande proporção de mata residual.

Os dados sobre os flebotomíneos foram obtidos a partir de capturas realizadas no período de maio de 2004 a maio de 2005 (quinze datas diferentes com intervalos de duas semanas até dois meses, entre 17 e 6 horas), na localidade do ramal do Granada. As datas das coletas entomológicas dependeram de questões técnicas, como acesso à área, disponibilidade de transporte, concordância das famílias envolvidas, e outras. Foram utilizadas 
armadilhas luminosas do tipo CDC conectadas a baterias de longa duração. Os flebotomíneos foram capturados nos ambientes silvestres (interior e borda de matas residuais) e antrópico, tanto intradomiciliar (dormitório ou cozinha) como peridomiciliar (galinheiros, chiqueiros ou varandas das casas). Os lotes escolhidos para a captura pertenciam a famílias com casos de leishmaniose tegumentar notificados até três meses antes do início do estudo entomológico ou durante a vigência do mesmo. Foram efetuadas no total seis coletas intradomiciliares, vinte $\mathrm{e}$ oito coletas peridomiciliares, seis coletas em borda de mata residual e uma coleta no interior de mata residual. Os insetos foram armazenados $\mathrm{a}-20^{\circ} \mathrm{C}$ até a identificação, utilizando-se a chave de Galati (2003) ${ }^{19}$.

\section{Análise Estatística}

A análise estatística foi feita utilizandose o software SPSS para Windows versão 13 (SPSS Inc., Illinois, USA). Duas ou mais proporções foram comparadas com o teste do $X^{2}$ ou $X^{2}$ com correção de Yates. Estimativas de odds ratio, com o respectivo intervalo de confiança de $95 \%$, foram calculadas para testar associações com tratamento, tendo como variável dependente a dose diária prescrita (adequada ou inadequada), como desfecho a resposta ao tratamento (sucesso ou falência) e como variável de ajuste o método diagnóstico (laboratorial ou clínico-epidemiológico), usando-se para isso o teste de $X^{2}$ de Mantel-Haenzel. As diferenças foram consideradas estatisticamente significantes quando o valor de $\mathrm{P}$ foi menor que 0,05 .

\section{Aspectos Éticos}

Este estudo faz parte de um estudo maior sobre a epidemiologia de doenças endêmicas na Amazônia em andamento no município de Acrelândia ${ }^{18}$, que foi aprovado pelo Comitê de Ética em Pesquisa do Instituto de Ciências Biomédicas da Universidade de São Paulo (parecer n. 318/CEP, de 19/07/2002).
Os autores, através de documentação assinada e enviada a RBE, declaram não existir nenhum tipo de conflito de interesses.

\section{Resultados}

\section{Características epidemiológicas da LT no município de Acrelândia}

Foram notificados 92 casos de leishmaniose tegumentar em moradores de Acrelândia, entre janeiro de 2001 e dezembro de 2004, sendo sete $(7,6 \%)$ LT recidivante (sendo todos com lesões cutâneas) e três (3,3\%) sem informação sobre o tipo de entrada. Portanto, esses 10 casos foram excluídos da análise por não ter sido possível verificar quando ocorreu a notificação inicial, utilizando-se para a análise dos dados apenas 82 fichas de notificação.

Aidade dos indivíduos com leishmaniose variou entre 2 e 69 anos (mediana $=23$ anos, média $=26,27$ anos, desvio-padrão $=15,58$ anos), sendo que $62(75,6 \%)$ casos eram do sexo masculino e somente $20(24,4 \%)$ do sexo feminino.

Dos 56 indivíduos com informação sobre o local de residência, $47(83,9 \%)$ residiam na zona rural e $9(16,1 \%)$ na zona urbana, com igual proporção de homens e mulheres afetados $(P=0,939)$. Em relação à ocupação principal, as mais freqüentes foram a de trabalhador autônomo $(\mathrm{n}=29 ; 35,4 \%)$, estudante $(\mathrm{n}=10 ; 12,2 \%)$, trabalhador agrícola $(\mathrm{n}=7 ; 8,5 \%)$ e do lar ( $\mathrm{n}=5 ; 6,1 \%$ ); em 19 fichas (23.2\%) não havia informação sobre ocupação. Como uma proporção considerável das fichas não apresentava dados sobre ocupação e local de moradia, não foi possível realizar uma análise mais detalhada sobre a associação entre atividade ocupacional, local de moradia e aquisição de leishmaniose.

A forma clínica dos casos novos de LT foi predominantemente cutânea (76 casos ou $92,7 \%$, dos quais 12 com lesões difusas) e raramente mucosa ou cutâneo-mucosa (4 casos; 4,9\%). Das 82 fichas avaliadas, 64 $(78,0 \%)$ apresentavam somente lesão cutânea única, doze $(14,7 \%)$ lesões cutâneas difusas, um $(1,2 \%)$ apenas lesão mucosa, três 
(3,7\%) lesões cutâneas e mucosas, e somente em dois casos (2,4\%) não havia informação sobre o tipo de lesão. O exame parasitológico direto foi realizado apenas em 59 pacientes, sendo positivo em todos aqueles testados ( $100 \%$ dos exames e $71,95 \%$ dos casos), e a reação intradérmica de Montenegro foi positiva em 47 dos 48 pacientes testados (98\% dos exames e $57,3 \%$ dos casos). O exame histopatológico da lesão (biópsia) foi realizado em 39 indivíduos (48,7\%); em 27 (69,2\%) foram encontradas formas de Leishmania spp.; 4 biópsias (10,3\%) foram consideradas "compatíveis" e somente 8 biópsias (20,5\%) foram consideradas "não-compatíveis". Dentre os 8 indivíduos com biópsia "não-compatível" com LT, seis apresentavam raspado da lesão positivo e os dois indivíduos restantes não tinham realizado nenhum outro exame complementar além do exame histopatológico. Dos 82 casos novos notificados no município, 20 (24,4\%) tiveram diagnóstico exclusivamente clínico-epidemiológico, e $2(2,4 \%)$ tiveram diagnóstico clínico com resultados laboratoriais negativos.

A droga escolhida como primeira opção de tratamento foi o antimonial pentavalente em todos os 81 casos que possuíam algum tipo de informação sobre tratamento na ficha de notificação. A dose prescrita foi de $15 \mathrm{mg} /$ $\mathrm{kg} /$ dia na maioria dos casos (51 pacientes; $62,2 \%$ do total de notificações); 2 pacientes $(2,4 \%)$ receberam dose inferior a $15 \mathrm{mg} /$ $\mathrm{kg} / \mathrm{dia} ; 3$ pacientes $(3,6 \%)$ receberam dose entre 16 e $20 \mathrm{mg} / \mathrm{kg} / \mathrm{dia}$ e 4 pacientes $(4,9 \%)$ receberam dose superior a $20 \mathrm{mg} / \mathrm{kg} / \mathrm{dia}$. Em 81 fichas com informação sobre tratamento, a terapia com antimonial pentavalente foi bem-sucedida em 58 indivíduos (71,6\%); 15 indivíduos (18,5\%) tiveram falência do tratamento inicial $\mathrm{e} o$ antimonial pentavalente foi utilizado em novo ciclo de tratamento; nos 8 casos restantes $(9,9 \%)$ não foi possível recuperar informações sobre a eficácia do tratamento inicial. Todos os 22 indivíduos sem confirmação laboratorial de LT receberam tratamento com antimonial pentavalente; dentre os 17 com evolução conhecida, 41,2\% apresentaram falência do tratamento farmacológico, sendo significantemente maior
$(\mathrm{P}=0,015)$ do que a encontrada no grupo de indivíduos com confirmação laboratorial de LT ( $14 \%$ de falência terapêutica). Esses dados sugerem que a falência terapêutica no primeiro grupo deve-se ao diagnóstico incorreto de LT.

Embora não se tenha a informação sobre a duração do tratamento de cada indivíduo, avaliou-se a adequação da dose prescrita à recomendação oficial do Ministério da Saúde para o tratamento de $\mathrm{LT}^{16}$. Dentre os 60 indivíduos com informação sobre o tipo de lesão e a dose prescrita, 14 $(23,3 \%)$ receberam dose diária menor do que a estabelecida no protocolo terapêutico recomendado pelo MS para o tratamento de LT, e $46(76,7 \%)$ receberam a dose diária recomendada. Entre os pacientes que receberam dose diária adequada, apenas 1 $(2,2 \%)$ apresentou falência do tratamento inicial. No entanto, entre os 14 indivíduos com dose diária inadequada, 9 (64,3\%) necessitaram novo ciclo de tratamento, sendo essa diferença estatisticamente significante (OR não-ajustado = 81,00; IC 95\% 8,42-778,69, $\mathrm{P}<0,0001)$. Considerando que o critério diagnóstico utilizado pode confundir a resposta ao tratamento (visto que pacientes com diagnóstico errôneo de LT não terão remissão das lesões), a análise da prevalência de falência terapêutica conforme a dose diária utilizada nos casos novos foi ajustada segundo o critério diagnóstico de LT empregado, e permaneceu estatisticamente significante $(\mathrm{P}<0,0001)$, resultando em um odds ratio sumário de MantelHaenzel de 67,80 (IC 95\% 7,99-575,30). Em outras palavras, o odds de necessitar de um segundo ciclo de tratamento entre aqueles que receberam dose diária de antimonial pentavalente abaixo das recomendações do MS foi 67,8 vezes o odds dos que receberam dose diária adequada, ajustando-se para o critério diagnóstico de LT utilizado.

\section{Fauna de flebotomíneos}

Foram coletados no total 40 espécimes de flebotomíneos, sendo 16 machos (40\%) e 24 fêmeas (60\%), de um total de 45 arma- 
dilhas em todo o estudo (eficiência geral de 0,89 flebotomíneo/armadilha). A fauna coletada é composta de 14 espécies pertencentes a três subtribos: Brumptomyiina (2,5\%), Lutzomyiina (20,0\%) e Psychodopygina $(77,5 \%)$. Houve predomínio da Nyssomyia antunesi (45\%) e da Nyssomyia whitmani (15\%) entre as espécies coletadas. A lista das espécies e números de espécimes capturados por ambiente encontra-se na Tabela 1.

No ambiente peridomiciliar de residências onde havia casos de LT foram coletados 22 espécimes (55\%) correspondendo a sete espécies, sendo que predominou a $N$. antunesi, com 13 espécimes (59,1\%). No ambiente intradomiciliar de um caso de LT (paciente idosa do sexo feminino, com pouca mobilidade) foi coletada apenas 1 (uma) fêmea de $N$. whitmani; essa espécie também foi encontrada (1 macho) no ambiente peridomiciliar da mesma casa. Nas bordas de matas residuais dos lotes, houve a maior riqueza de espécies (09) e a captura de 16 espécimes, com ligeiro predomínio da N. antunesi e N. whitmani, ambas respondendo por $25 \%$ dos espécimes coletados. Um macho e uma fêmea de Trichophoromyia ubiquitalis, de Evandromyia walkerie de Sciopemyia servulolimai também foram capturados no peridomicílio. N. antunesi foi a única espécie encontrada no interior da mata (em uma única coleta).

Tabela 1 - Número de espécimes de flebotomíneos coletados com armadilhas automáticas luminosas, tipo CDC, na zona rural de Acrelândia, AC (Ramal do Granada), segundo subtribo, gênero, espécie, sexo e ambiente de coleta.

Table 1 - Number of specimens collected with CDC light traps in rural areas of Acrelandia, AC (Ramal do Granada), according to sub tribe, genus, species, sex and type of environment.

\begin{tabular}{|c|c|c|c|c|c|c|c|c|}
\hline \multirow{2}{*}{ Subtribo } & \multirow{2}{*}{ ESPÉCIE } & \multicolumn{2}{|c|}{ PD } & \multirow{2}{*}{$\begin{array}{c}\text { ID } \\
\text { + } \\
\end{array}$} & \multicolumn{2}{|c|}{ BM } & \multirow{2}{*}{$\begin{array}{c}\mathrm{IM} \\
\hat{0}\end{array}$} & \multirow{2}{*}{$\begin{array}{c}\text { Total } \\
\hat{\jmath} / 9(\%) \\
\end{array}$} \\
\hline & & 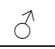 & 우 & & $\hat{0}$ & $q$ & & \\
\hline Brumptomyiina & Brumptomyia sp. & - & 1 & - & - & - & - & $1(2,5)$ \\
\hline \multirow{6}{*}{ Lutzomyiina } & $\begin{array}{l}\text { Evandromyia saulensis (Floch \& } \\
\text { Abonnenc, 1944) }\end{array}$ & - & - & - & - & 1 & - & $1(2,5)$ \\
\hline & $\begin{array}{l}\text { Evandromyia bacula (Martins, Falcão \& } \\
\text { Silva, 1965) }\end{array}$ & - & - & - & 1 & - & - & $1(2,5)$ \\
\hline & Evandromyia walkeri (Newstead, 1914) & 1 & 1 & - & - & - & - & $2(5,0)$ \\
\hline & $\begin{array}{l}\text { Lutzomyia sherlocki (Martins, Falcão \& } \\
\text { Silva, 1971) }\end{array}$ & - & - & - & - & 1 & - & $1(2,5)$ \\
\hline & $\begin{array}{l}\text { Sciopemyia servulolimai (Damasceno } \\
\text { \& Causey, 1945) }\end{array}$ & 1 & 1 & - & - & - & - & $2(5,0)$ \\
\hline & $\begin{array}{l}\text { Sciopemyia sordellii (Shannon \& Del } \\
\text { Ponte, 1927) }\end{array}$ & - & - & - & 1 & - & - & $1(2,5)$ \\
\hline \multirow{7}{*}{ Psychodopygina } & Nyssomyia antunesi (Coutinho, 1939) & 6 & 7 & - & - & 4 & 1 & $18(45,0)$ \\
\hline & $\begin{array}{l}\text { Nyssomyia whitmani (Antunes \& } \\
\text { Coutinho, 1939) }\end{array}$ & 1 & - & 1 & - & 4 & - & $6(15,0)$ \\
\hline & Psychodopygus davisi (Root, 1934) & - & - & - & 1 & 1 & - & $2(5,0)$ \\
\hline & $\begin{array}{l}\text { Trichophoromyia brachipyga } \\
\text { (Mangabeira, 1942) }\end{array}$ & 1 & - & - & - & - & - & $1(2,5)$ \\
\hline & $\begin{array}{l}\text { Trichophoromyia ubiquitalis } \\
\text { (Mangabeira, 1942) }\end{array}$ & 1 & 1 & - & - & - & - & $2(5,0)$ \\
\hline & Trichophoromyia sp. & - & - & - & - & 1 & - & $1(2,5)$ \\
\hline & $\begin{array}{l}\text { Viannamyia furcata (Mangabeira, } \\
\text { 1941) }\end{array}$ & - & - & - & 1 & - & - & $1(2,5)$ \\
\hline TOTAL & & 11 & 11 & 1 & 4 & 12 & 1 & $40(100,0)$ \\
\hline
\end{tabular}

Nota: $\hat{\delta}=$ macho,,+ = fêmea, $\mathrm{PD}=$ peridomiciliar, $\mathrm{ID}=$ intradomiciliar, $\mathrm{BM}=$ borda de mata residual, $\mathrm{IM}=$ interior de mata residual.

Note: $\widehat{\delta}=$ male, $q=$ female, $P D=$ peri-domiciliary, $I D=$ intra-domiciliary, $B M=$ border of residual forest, $I M=$ interior of residual forest. 


\section{Discussão}

As características clínico-epidemiológicas da LT observadas em Acrelândia (predomínio da forma cutânea e acometimento de indivíduos adultos do sexo masculino) são similares ao que foi descrito no Acre $^{2,20}$, Maranhão ${ }^{21}$, Bahia ${ }^{22}$ e Amazonas ${ }^{23}$, e indicam predomínio de transmissão extradomiciliar. No entanto, a ocorrência de casos em crianças pequenas sugere estar ocorrendo também transmissão intra e/ou peridomiciliar em Acrelândia, embora com menor freqüência, semelhante ao tipo de transmissão relatada na Bahia ${ }^{22}$. Chagas e colaboradores $(2006)^{23}$ conseguiram associar a transmissão de LT com atividades profissionais florestais ou peri-florestais numa área de mineração no Amazonas. Esse tipo de associação não é factível no presente estudo, já que o município não tem uma clara definição entre zona rural e urbana, e há constantes deslocamentos dos moradores entre o núcleo urbano e propriedades agrícolas próximas, mesmo entre profissionais com atividades predominantemente não-rurais.

Considerando-se as características da região estudada (perímetro extenso com difícil acesso da população aos meios de transporte, pequeno número de unidades notificadoras, desconhecimento da população a respeito da doença), é de se esperar que esteja ocorrendo subnotificação de casos, principalmente da zona rural. Além disso, o pequeno número de recidivas também pode ser devido à subnotificação e/ou intervalo de seguimento curto. Portanto, o número de casos de LT pode ser maior do que o notificado.

Em relação aos exames complementares utilizados, foram empregados mais freqüentemente o exame direto do raspado das lesões e a reação intradérmica de Montenegro, ambos com boa positividade, de fácil execução e baixo custo. Finalmente, o terceiro exame complementar utilizado em Acrelândia foi o histopatológico, que, em muitos casos, foi inconclusivo, além de ser um método de maior complexidade e custo elevado em relação aos demais. Por outro lado, uma proporção expressiva de notificações $(24,4 \%)$ baseou-se no diagnóstico clínico-epidemiológico apenas, que em determinadas situações (especialmente em áreas remotas da região amazônica) pode ser o único critério diagnóstico ao alcance do serviço básico de saúde.

Os dados sobre resposta ao tratamento com antimoniais pentavalente indicaram haver grande freqüência de falência terapêutica quando a dose diária esteve abaixo das recomendações do Ministério da Saúde, e quando o diagnóstico de LT baseou-se exclusivamente em critérios clínico-epidemiológicos, sem utilização de algum exame laboratorial. Esses dados sugerem primeiramente que parte das notificações sem confirmação laboratorial possa, na verdade, não corresponder à doença leishmaniótica, e demonstram a importância de se confirmar o diagnóstico laboratorialmente. Em segundo lugar, fica evidente a necessidade de se utilizar a dose diária de antimoniais pentavalente $(\mathrm{em} \mathrm{mg} / \mathrm{kg} / \mathrm{dia})$ recomendada pelo Ministério da Saúde ${ }^{16}$ para o sucesso da terapia. A não-utilização dos recursos laboratoriais existentes e a utilização de doses inadequadas levam não só ao desperdício de recursos financeiros (quer por tratar nãodoentes ou por necessidade de um segundo tratamento) como à exposição do paciente a um risco desnecessário, visto que a terapia com antimoniais pentavalente tem uma série de efeitos colaterais, alguns dos quais graves, como arritmias cardíacas ${ }^{16}$.

Houve algumas limitações no uso dos dados epidemiológicos provenientes das fichas de notificação, uma vez que dados como nome, local de moradia, profissão, tipo de evolução, local provável de infecção e deslocamentos estavam ausentes ou incompletos em grande parte das fichas. Entretanto, foi possível recuperar dados importantes sobre diagnóstico e tratamento em 97,2\% e 73,22\% das fichas, respectivamente, bem como avaliar a freqüência de falência terapêutica em $89 \%$ dos casos, permitindo não só a identificação de problemas de diagnóstico e tratamento da LT no município, mas também a elaboração de recomendações baseadas 
em evidências colhidas pelo próprio serviço de saúde por meio das fichas de notificação, indicando a utilidade desse tipo de dado. Os resultados entomológicos obtidos, embora preliminares, mostram predomínio de espécies de Psychodopygina (77,5\%), grupo no qual se concentram os vetores de LT e, neste, o do gênero Nyssomyia $(77,4 \%)$. A presença de $N$. whitmani em ambientes intra e peridomiciliares e borda de mata sugere que esta espécie possa ser um importante vetor de LT na zona rural do município, levantando a hipótese de uma possível circulação na área de estudo de L. (V.) shawi (Lainson et al., 1989), além da L. braziliensis, o agente da LT de mais ampla distribuição no Brasil ${ }^{6,7}$.

Para Nyssomyia antunesi, encontra-se descrita na literatura ${ }^{24}$ infecção natural por promastigotas em três espécimes desta espécie no Pará, sugerindo infecção pelo gênero Leishmania, sem, no entanto, identificar a espécie do parasita. Também suspeita-se de sua participação como vetor de $L$. (V.) lindenbergi (Silveira et al., 2002), em foco identificado em Belém ${ }^{6}$. Assim, é possível que este flebotomíneo esteja atuando como vetor de LT em Acrelândia, principalmente nos ambientes peridomiciliares e bordas de matas residuais. Outra espécie que pode ser um potencial vetor na região éa T. ubiquitalis, que foi incriminada como transmissora de $L$. (V.) lainsoni (Silveira et al, 1987) no Pará ${ }^{25}$, e que foi encontrada no presente estudo no ambiente peridomiciliar de uma residência com três casos de LT. A $L$. (V.) lainsoni também foi descrita nas florestas do Peru e da Bolívia ${ }^{26,27}$. Recentemente foi publicada uma série de 50 casos de LT provenientes de sete municípios próximos de Acrelândia, onde se confirmou por meio de cultura e testes moleculares a circulação de $L$. (V.) lainsoni nessa área, além de L. (V.) braziliensis e L. (V.) guyanensis ${ }^{20}$. Face a esta primeira identificação de T. ubiquitalis na área de estudo e da ocorrência de $L$. (V.) lainsoni em áreas vizinhas, a provável circulação dessa espécie de Leishmania na região de Acrelândia deve ser levada em consideração. As espécies da subtribo Lutzomyiina não têm sido associadas à transmissão de Leishmania, com exceção de Lutzomyia sherlocki, que pertence ao subgênero Tricholateralis (Galati, 1995). Esse subgênero é dotado de algumas espécies antropofílicas que têm importância na transmissão de LT na América Latina ${ }^{28}$.

Embora o número de espécimes capturados (40) no total dos ambientes amostrados tenha sido pequeno, chama atenção a riqueza de espécies coletadas (14) em uma mesma localidade, disposta ao longo de uma estrada de 18 km de extensão. Estudos em Rio Branco e Cruzeiro do Sul realizados na década de 70 identificaram um número maior de espécies (46 e 28 respectivamente) ${ }^{4}$; no entanto, o tamanho da área amostrada e o total de espécimes coletados foram maiores do que presente estudo. A riqueza de espécies na área rural de Acrelândia também está refletida no ambiente peridomiciliar (9 espécies), podendo ser um indicativo de um processo de adaptação dessas populações às mudanças rápidas da paisagem nativa, incluindo espécies transmissoras de LT. Além disso, os resultados desse estudo entomológico preliminar no município de Acrelândia estão de acordo com as espécies identificadas como circulantes nessa área do estado ${ }^{20} \mathrm{e}$ também sugerem a circulação de outras espécies além das já descritas. O presente estudo também contribuiu com a identificação de duas espécies de flebotomíneos ainda não citadas para o Acre, T. ubiquitalis e S. servulolimai.

\section{Conclusões}

As características epidemiológicas da LT em Acrelândia parecem ser semelhantes a outros municípios da região amazônica e extra-amazônica. Embora a utilização de dados secundários possa apresentar problemas de interpretação (visto não ser possível controlar a qualidade dos dados obtidos), esta abordagem possibilitou identificar condutas inadequadas no Sistema de Saúde avaliado que podem estar contribuindo para a manutenção da endemia no município de Acrelândia, notadamente o diagnóstico de LT baseado em critérios clínicos apenas, e o tratamento farmacológico com doses inapropriadas.

Não foi possível neste estudo isolar e identificar qual(is) Leishmania spp. está(ão) 
associada(s) à doença no município. Entretanto, uma primeira exploração da fauna de flebotomíneos do local foi capaz de identificar uma grande diversidade de espécies com pelo menos três potenciais vetores. A presença de N. whitmani [um dos mais importantes vetores da LT, tanto da Leishmania (Viannia) braziliensis como também da Leishmania (V.) shawi], Nyssomyia antunesi [suspeito de transmitir a Leishmania (V.) lindenbergi Silveira et al. 2002] e Trichophoromyia ubiquitalis [vetor incriminado de transmitir a Leishmania (V.) lainsoni] em ambiente antrópico da zona rural de Acrelândia pode indicar que essas espécies vetoras ou potencialmente vetoras estejam se ambientando em áreas agrícolas desmatadas próximas a matas residuais, uma condição freqüente na Amazônia atual devido à expansão das fronteiras agrícolas no Norte do País. Esses dados preliminares são úteis para direcionar estudos futuros de identificação do parasita, além dos já existentes, e também aumentar o conhecimento da fauna local, com a documentação da ocorrência de duas espécies nunca antes identificadas no Acre.

\section{Agradecimentos}

Os autores expressam seus agradeci- mentos a Sebastião Bocalom Rodrigues, Damaris de Oliveira, Nésio Mendes de Carvalho (Prefeitura Municipal de Acrelândia) e à equipe de Saúde de Acrelândia pelo apoio de infraestrutura e epidemiológico; aos membros da Secretaria de Estado da Saúde e Saneamento do Acre em Rio Branco e em Acrelândia pelo excelente apoio logístico; aos moradores de Acrelândia que participaram do estudo; a Adamílson Luís de Souza pela assistência durante todo o trabalho de campo, e a Edna Fátima Maria Bueno pelo processamento laboratorial dos flebotomíneos.

\section{Colaboradores}

M. da Silva-Nunes e E.A.B. Galati planejaram as coletas entomológicas; M. da Silva-Nunes executou todas as coletas de flebotomíneos na área endêmica, analisou os dados epidemiológicos e redigiu o artigo; C.E. Cavasini e N.S. Silva coletaram e consolidaram os dados epidemiológicos, C.E. Cavasini colaborou na análise dos dados epidemiológicos e na redação do artigo; E.A.B. Galati realizou a identificação dos flebotomíneos, analisou os dados entomológicos e supervisionou a redação do artigo, que foi lido e aprovado por todos os autores.

\section{Referências}

1. Ministério da Saúde, Secretaria de Vigilância em Saúde. Leishmaniose Tegumentar Americana, Distribuição de casos confirmados por Unidade Federada, Brasil 1980-2005. http://www.portal.saude.gov.br/portal/SVS [Acessado em 25/05/2006].

2. Silva NS, Viana AB, Cordeiro JC, Cavasini CE. Leishmaniose tegumentar americana no Estado do Acre, Brasil. Rev Saúde Pública 1999; 33: 554-9.

3. Arias JR, Freitas RA. On the vectors of cutaneous leishmaniasis in the Central Amazon of Brazil. 3: Phlebotomine sandfly stratification in "terra firme" forest. Acta Amazônica 1982; 12: 599-609.

4. Arias JR, Freitas RA. Flebótomos da Amazônia Central do Brasil. I. Resultados obtidos das capturas feitas em isca humana e equina (Diptera: Psychodidae). Acta Amazônica 1977; 7: 507-27.
5. Lainson R, Shaw JJ, Ward RD, Ready PD, Naiff RD. Leishmaniasis in Brazil. XIII. Isolation of Leishmania from armadillos (Dasypus novemcinctus), and observations on the epidemiology of cutaneous leishmaniasis in north Pará state. Trans R Soc Trop Med Hyg 1979; 3: 239-42.

6. Lainson R, Shaw JJ. New World Leishmaniasis - the neotropical Leishmania species. In: Cox FEG, Kreier JP, Wakelin D (org.). Topley and Wilson's Microbiology and Microbial Infections. London: Arnold; 2005. p. 313-49.

7. Rangel EF, Lainson R, Souza AA, Ready P, Azevedo ACR. Variation between geographical populations of Lutzomyia (Nyssomyia) whitmani (Antunes \& Coutinho, 1939) sensu lato (Diptera: Psychodidae: Phlebotominae) in Brazil. Mem Inst Osvaldo Cruz 1996; 91: 43-50. 
8. Shaw JJ, Ishikawa EA, Lainson R, Braga RR, Silveira FT. Cutaneous leishmaniasis of man due to Leishmania (Viannia) shawi Lainson, de Souza, Póvoa, Ishikawa \& Silveira in Pará State, Brazil. Ann de Parasitol Hum Comp 1991; 66: 243-6.

9. da Costa SM, Cechinel M, Bandeira V, Zannuncio JC, Lainson R, Rangel EF. Lutzomyia (Nyssomyia) whitmani s.l. (Antunes \& Coutinho, 1939) (Diptera: Psychodidae: Phlebotominae): geographical distribution and the epidemiology of American cutaneous leishmaniasis in Brazil-mini-review. Mem Inst Oswaldo Cruz 2007; 102:149-53.

10. Feitosa MAC, Castellón EG. Fauna de flebotomíneos (Diptera: Psychodidae) em fragmentos florestais ao redor de conjuntos habitacionais na cidade de Manaus, Amazonas, Brasil. II. Estratificação horizontal. Acta Amazônica 2004; 34:121-7.

11. Ready PD, Lainson R, Shaw JJ. The ecology of Lutzomyia umbratillis Ward \& Fraiha, 1977 (Diptera: Psychodidae) the major vector to man of Leishmania braziliensis guyanensis northeast amazonian in Brazil. Bull Entomol Res 1986; 76: 21-40.

12. Azevedo ACR, Luz SLB, Vilela ML, Rangel EF. Studies on the sandfly fauna of Samuel Ecological Station, Porto Velho Municipality, Rondônia State, Brazil. Mem Inst Osvaldo Cruz 1993; 88: 509-12.

13. Datasus, Ministério da Saúde. http://www.datasus.gov. br [Acessado em 27/12/2007].

14. Sistema Estadual de Informações Ambientais (SEIAM), Governo do Estado do Acre. http://www.seiam.ac.gov.br [Acessado em 24/05/2006].

15. Atlas do Desenvolvimento Humano no Brasil, Programa das Nações Unidas para o Brasil (PNUD). http://pnud. org.br/atlas [Acessado em 15/12/2006].

16. Ministério da Saúde, Fundação Nacional de Saúde. Manual de controle da Leishmaniose Tegumentar Americana. Brasília: Ministério da Saúde, Fundação Nacional de Saúde; 2000.

17. Gontijo B, de Carvalho M de L. American Cutaneous Leishmaniasis. Rev Soc Bras Med Trop 2003; 36: 71-80.

18. Silva-Nunes M, Malafronte RS, Luz BA, Souza EA, Martins LC, Rodrigues SG, et al. The Acre Project: the epidemiology of malaria and arthropode-borne virus infections in a rural Amazonian population. Cad Saúde Pública 2006; 22: 1325-34.
19. Galati EAB. Morfologia, terminologia de adultos e identificação dos táxons da América. In: Rangel EF, Lainson R, organizadores. Flebotomíneos do Brasil, Rio de Janeiro: Editora Fiocruz; 2003. p. 53-175.

20. Tojal da Silva AC, Cupolillo E, Volpini AC, Almeida R, Romero GA. Species diversity causing human cutaneous leishmaniasis in Rio Branco, state of Acre, Brazil. Trop Med Int Health 2006; 11: 1388-98.

21. Martins LM, Rebêlo JMM, Santos MCFV, Costa JML, Silva AR, Ferreira LA. Ecoepidemiologia da leishmaniose tegumentar no Município de Buriticupu, Amazônia do Maranhão, Brasil, 1996 a 1998. Cad Saúde Pública 2004; 20: 735-43.

22. Ampuero J, Macêdo V, Marsden P. Características clínicas da leishmaniose tegumentar em crianças de 0 a 5 anos em uma área endêmica de Leishmania (Viannia) braziliensis. Rev Soc Bras Med Trop 2006; 39: 22-6.

23. Chagas AC, Pessoa FAC, Medeiros JF, Py-Daniel V, Mesquita EC, Balestrassi DA. Leishmaniose Tegumentar Americana (LTA) em uma vila de exploração de minérios - Pitinga, município de Presidente Figueiredo, Amazonas, Brasil. Rev Bras Epidemiol 2006; 9: 186-92.

24. Ryan L, Silveira FT, Lainson R, Shaw JJ. Leishmanial infections in Lutzomyia longipalpis and Lu. antunesi (Diptera, Psychodidae) on the island of Marajó, Pará State, Brazil. Trans R Soc Trop Med Hyg 1984; 78: 547-8.

25. Silveira FT, Souza AAA de, Lainson R, Shaw JJ, Braga RR, Ishikawa EAY. Cutaneous leishmaniasis in the Amazon region, natural infection of the sandfly Lutzomyia ubiquitalis (Psychodidae: Phlebotominae) by Leishmania (Viannia) lainsoni in Pará State, Brazil. Mem Inst Oswaldo Cruz 1991; 86: 127-30.

26. Lucas CM, Franke ED, Cachay MI, Tejada A, Carrizales D, Kreutzer RD. Leishmania (Viannia) lainsoni, first isolation in Peru. Am J Trop Med Hyg 1994; 51: 533-7.

27. Martinez E, Le Pont F, Mollinedo S, Cupolillo E. A first case of cutaneous leishmaniasis due to Leishmania (Viannia) lainsoni in Bolivia. Trans R Soc Trop Med Hyg 2001; 95: 375-7.

28. Young DG, Duncan MA. Guide to the identification and geographical distribution of Lutzomyia sand flies in Mexico, the West Indies, Central and South America (Diptera: Psychodidae). Mem Amer Entomol Inst 1994; 54: 1-881.
Recebido em: 29/01/07

Versão final reapresentada em:08/03/08 Aprovado em: 19/03/08 